\title{
Acute tacrolimus nephrotoxicity in kidney transplanted patients - from kidney biopsy to urinary markers of acute kidney injury: a case report
}

Angelica Gramaticu ${ }^{1}$, Daniela Constantinescu ${ }^{2,3}$, Adina Covic ${ }^{1}$, Dimitrie Siriopol ${ }^{1,4}$, Raluca Dumea ${ }^{1,4}$, Simona Hogaș ${ }^{1,4}$, Irina-Draga Căruntu ${ }^{5}$, Corina Cianga ${ }^{2,3}$, Adrian Covic $^{1,4}$, Petru Cianga*,2,3

${ }^{1}$ Department of Nephrology, "Dr. Cl Parhon" Hospital, lasi, Romania; 'Department of Immunology, University of Medicine and Pharmacy "Grigore T. Popa" lasi; ${ }^{3}$ Laboratory of Immunology and Genetics "Sf. Spiridon" Hospital, lasi, Romania; ${ }^{4}$ Department of Nephrology, "Grigore T. Popa" University of Medicine and Pharmacy, lași, Romania; ${ }^{5}$ Department of Morphofunctional Sciences, "Grigore T. Popa" University of Medicine and Pharmacy, lași, Romania

\begin{abstract}
Calcineurin inhibitors (CNIs) play a major role in kidney transplant immunosuppressive regimens, but they also may cause acute and chronic kidney toxicity, which is an important cause of long-term graft failure if not recognized and treated promptly. Therapeutic approaches are different and sometimes even opposite in acute graft dysfunction, requiring detailed differential diagnosis. Current guidelines consider the renal biopsy to have the highest specificity and sensitivity in the diagnosis and correct therapy guidance of acute graft dysfunction. However, the renal biopsy is an invasive and expensive method that predisposes to complications. In the last decade, a number of studies were focused on the urinary levels of various biomarkers like kidney injury molecule1 (KIM-1), neutrophil gelatinase associated lipocalin (NGAL), interferon induced protein-10 (IP-10) or cystatin C (CysC) as potentially valuable non-invasive methods of allograft pathology diagnosis. We report the case of a 27 year-old male who underwent a kidney transplant and for which the urinary measurement of these biomarkers proved extremely useful in diagnosing the acute tacrolimus nephrotoxicity, which further allowed a correct therapeutic approach.
\end{abstract}

Keywords: KIM-1, NGAL, CysC, IP-10, kidney transplant

\section{Introduction}

Renal transplantation is the preferred method of renal replacement therapy due to the significantly increased quality of life and effectiveness [1-2]. Unfortunately, allograft recipients are facing multiple complications

Received: July 2015; Accepted after review: August 2015; Published: September 2015.

${ }^{*}$ Corresponding author: Prof. Dr. Petru Cianga, MD, $\mathrm{PhD}$, Grigore T. Popa University of Medicine and Pharmacy lasi, 16, Universitatii Street, 700115, lasi, Romania

E-mail: petrucianga@hotmail.com that could lead to graft loss. Acute renal graft dysfunction can be caused by acute rejection $(A R)$, nephrotoxicity of calcineurin inhibitors (CNIs), acute tubular necrosis (ATN) or ischemia/reperfusion lesions. Nevertheless, the major problem is that all of them present with the same biological features: increasing serum creatinine levels that are not always associated with clinical signs like oliguria or fever [3].

CNIs play an essential part in the standard immunosuppressive regimens in combination with mycophenolate mofetil (a reversible 
inhibitor inosine monophosphate dehydrogenase - IMPDH) and corticosteroids [4], but they also may cause acute and chronic kidney toxicity [5], which is an important cause of long-term graft failure if not recognized and treated promptly. The therapeutic approaches are different and quite opposite in AR and CNIs nephrotoxicity, the first requiring immunosuppression augmentation while in the second case it is necessary to lower the CNIs doses. Therefore, a rapid and correct diagnosis is crucial.

Currently, renal biopsy is considered to have the highest specificity and sensitivity in the diagnosis and correct therapy guidance of acute graft dysfunction [6]. However, the renal biopsy is an invasive and expensive method that predisposes to complications. Consequently, the availability of a noninvasive test with high sensitivity and specificity is of maximum importance. In the last decade, a number of studies focused on the urinary levels of various biomarkers as potentially valuable non-invasive methods of diagnosis of the allograft pathology.

Neutrophil gelatinase-associated lipocalin (NGAL) known also as lipocalin-2 is secreted by hepatocytes, gastrointestinal tract cells and kidney tubular cells and is excreted in the urine through the epithelial cells of Henle loop [7]. NGAL value as an early tool of predicting acute kidney injury (AKI) was proven in contrast-induced $\mathrm{AKI}, \mathrm{AKI}$ in critical care and even cardiac-surgery associated AKI [8-9].

Kidney injury molecule 1 (KIM-1) is a type 1 transmembrane protein, that is specifically up-regulated in the proximal tubular cells after ischemic and nephrotoxic acute kidney injury [10-11]. In a prospective pilot study in adults undergoing on-pump cardiac surgery, urinary KIM-1 displayed the best diagnostic performance for detection of $\mathrm{AKI}$ when compared to NGAL and interleukin-18 (IL-18), a marker also considered as a valuable early predictor of acute kidney injury [12-13].

Studies focusing on Interferon-gamma induced protein 10 (IP-10), a reduced dimension chemokine synthesized by monocytes, endothelial cells and fibroblasts in response to IFN-gamma [14] revealed that the urinary levels of IP-10 are high during episodes of $A R$ and polyoma $B K$ virus infection, but are not elevated in calcineurin inhibitors toxicity and stable patients [15].

Cystatin C (CysC) is a protease inhibitor that is freely filtered through the glomerular membrane and then reabsorbed by tubular cells, maintaining a constant serum level dependent on the glomerular filtration rate. It was suggested that $\mathrm{Cys} C$ urinary levels could allow accurate detection of tubular injury [16].

We report the case of a 27 year-old male who underwent a kidney transplant and for which the urinary measurement of these biomarkers proved extremely useful in diagnosing acute tacrolimus (Tac) nephrotoxicity, which further allowed a correct therapeutic approach.

\section{Case report}

In May 2015, a 27 year-old male, with end stage renal disease secondary to type 2 membranoproliferative glomerulonephritis, on hemodialysis since June 2011, was admitted in our unit for living donor renal transplantation. As important associated pathologies we mention congenital atrial septal defect that required surgery in August 2012, and hypothyroidism that imposed hormone replacement therapy.

The living related donor was his 50 years old mother, with the same blood type (A II), sharing a haplotype match in the Human Leukocyte Antigen (HLA) compatibility system $\left(\mathrm{HLA}-\mathrm{A}^{*} 03, \mathrm{~B}^{*} 07\right.$ and $\left.\mathrm{DRB} 1^{*} 07\right)$ with our patient. Clinical and biological evaluation revealed no contraindications for the surgical procedure that was conducted without any surgical incidents.

Induction therapy was started the day of the surgery, using basiliximab (an antiinterleukin 2 receptor monoclonal antibody, 20 $\mathrm{mg}$ ) followed by triple immunosuppressive regimen consisting of tacrolimus (0.15 $\mathrm{mg} / \mathrm{kg} /$ day in two doses), mycophenolate mofetil (2g/day in two doses) and corticosteroids (Metilprednisolon $1 \mathrm{~g} /$ day-3 days, followed by oral prednisone $0.5 \mathrm{mg} /$ day).

After the procedure, the patient presented good immediate graft function with $16,500 \mathrm{ml}$ diuresis in the first day, followed by $6,500 \mathrm{ml}$ in the second day. The second day, the 
biological investigations revealed a decrease of the serum creatinine from $12.9 \mathrm{mg} / \mathrm{dl}$ before transplantation to $4.4 \mathrm{mg} / \mathrm{dl}$. The white cell blood count was high $\left(12,800 / \mathrm{mm}^{3}\right)$, but with a normal platelet count, $9.7 \mathrm{~g} / \mathrm{dl}$ hemoglobin level and normal electrolytes. According to the transplant protocol, abdominal ultrasound was performed that showed correct vascularization of the renal allograft, no signs of ureteral obstructions and peritoneal liquid in the Morrison space.

However, the third day marked a severe diuresis decrease from $6,500 \mathrm{ml}$ to $770 \mathrm{ml}$, accompanied by an increase of the white cell blood count to $30,200 / \mathrm{mm}^{3}$, with a high sedimentation rate $(60 \mathrm{~mm} / \mathrm{h})$ but without clinical signs of systemic infection. Procalcitonin, a marker that appears early in sepsis and is more specific to bacterial infections than the sedimentation rate [17-18], was slightly elevated $(1.46 \mathrm{ng} / \mathrm{ml}$, normal range $0.02-0.5 \mathrm{ng} / \mathrm{ml}$ ). A new abdominal ultrasound check was performed, showing no alterations in the kidney vascularization. Tac blood quantification revealed high levels (46 $\mathrm{ng} / \mathrm{ml}$, while renal transplantation guidelines accept levels between $7-10 \mathrm{ng} / \mathrm{ml}$ ).

Thus, the following suspicions were raised: acute CNIs nephrotoxicity and systemic infection. As a consequence several therapeutic strategies were adopted: lowering Tac doses from $0.2 \mathrm{mg} /$ day to $0.04 \mathrm{mg} /$ day, large spectrum antibiotics and Thymoglobulin (rabbit anti-human thymocyte immunoglobulin) administration to prevent a potential acute rejection, consequence to low Tac levels. Under the new therapeutic strategy, the white blood cell count and Tac levels decreased, but the serum creatinine levels augmented to 8.6 $\mathrm{mg} / \mathrm{dl}$ and the patient maintained a low urine output (380 $\mathrm{ml}$ and $195 \mathrm{ml}$ ).

According to KDIGO guidelines, the differential diagnosis imposed a renal allograft biopsy, which was performed in the fifth day. Simultaneously, the X-MAP technology was employed for the measurement of the four previously mentioned urinary markers: IP-10 (CXCL10), CysC, NGAL and KIM-1. We have used the Magnetic Luminex Performance Assay multiplex kit (R\&D Systems, MN, USA), based on color magnetic particles, coated with antibodies targeting the analytes of interest.
The reading was performed with a Luminex device manufactured by GenProbe (USA), using the Xponent software (GenProbe) for data acquisition and the MatchIT software for interpreting the results.

Urinary measurements revealed high levels of NGAL $(79,500.1 \mathrm{pg} / \mathrm{ml}$ - reference value $6,372.5 \mathrm{pg} / \mathrm{ml})$, cystatin C $(41,623.174$ $\mathrm{pg} / \mathrm{ml}$ - reference value $7,202.75 \mathrm{pg} / \mathrm{ml}$ ) and KIM-1 (397.459 pg/ml-reference value 114.5 $\mathrm{pg} / \mathrm{ml})$, but a normal IP-10 value $(510.64 \mathrm{pg} / \mathrm{ml}$ - reference value $1,474.5 \mathrm{pg} / \mathrm{ml}$ ).

The renal biopsy showed tubular injury, revealing minimal changes of the glomerular membrane, a sever dilatation of the renal tubular component, with epithelium flattening, loss of the striated border in the proximal convoluted tubules and minimal interstitial edema, all that suggesting acute CNIs nephrotoxicity.

As a consequence of these biological and histological findings, we have replaced Tac with low doses of Cyclosporine A (1 mg/day, in two daily doses) and we have added Verapamil and Pentoxifilin as vasodilators, considering the afferent arterioles vasoconstriction pivotal role in acute $\mathrm{CNIs}$ nephrotoxicity. Even if in this situation m-Tor inhibitors should have been the first choice, we have opted for Cyclosporine A (CsA) due to the high proteinuria (3.82 urinary proteincreatinine ratio).

The kidney function recovery was slow, oliguria and high creatinine levels were maintained for seven weeks, requiring eight sessions of hemodialysis. At discharge the patient had a normal diuresis $(2,500 \mathrm{ml} /$ day $)$ and a serum creatinine of $5.4 \mathrm{mg} / \mathrm{dl}$, which decreased to $1.54 \mathrm{mg} / \mathrm{dl}$ in two weeks.

\section{Discussions}

CNIs include CsA and Tac, two distinct molecules that act alike by inhibiting calcineurin and preventing gene expression responsible for lymphocytes $T$ activation. They also induce TGF $\beta$ expression and thereby inhibit IL-2 expression and are metabolized by the means of the cytochrome P450 III enzymatic system (CYP3A4) [19]. Randomized controlled trials showed Tac 
superiority over CsA in regard to graft function, graft survival and preventing acute rejection [20-22].

Acute nephrotoxicity may appear with variable clinical, histological and laboratory changes and are usually, but not always, dose-dependent. The main injuries are secondary to afferent arteriolar vasoconstriction. Thus, the renin-angiotensin system is activated and the sympathetic activity is increased, leading to an unbalanced vasodilator/vasoconstrictor ratio of eicosanoids that promotes renal vasoconstriction [23]. The clinician should suspect $\mathrm{CNIs}$ acute nephrotoxicity when confronting oliguria and high serum creatinine levels that cannot be explained by other causes. Therefore, the diagnosis is rather an exclusion process [24].

AR episodes and CNIs nephrotoxicity are major predictors of tubular and interstitial fibrosis leading to irreversible graft dysfunction. Some allografts may never recover their function after an episode of AR or acute CNIs nephrotoxicity, while those who do will have a poor long-term survival as compared to a graft not facing these problems [25].

Therefore, markers of acute kidney injury that could distinguish between these complications are extremely valuable. Several literature data supported our choice.

In a prospective multicenter observational cohort study of deceased donor allograft recipients, urinary $\mathrm{Cys} \mathrm{C} / \mathrm{creatinine}$ ratio presented high values in delayed graft function [26].

A case report suggested that KIM-1 expression, identified by immunohistochemistry on allograft biopsies, could precede morphological changes in kidney tubular cells [27]. In another study that included living donor transplant recipients, serial urinary KIM-1 measurement revealed that high levels of the protein can predict delayed graft function [28].

Furthermore, in a study that included 69 transplanted patients, KIM-1 expression on kidney allografts biopsies was strongly positive in the chronic active antibody mediated rejection, but weakly positive in the group without rejection or injury due to immunosuppressant toxicity, thus suggesting that $\mathrm{KIM}-1$ expression is associated with tubular cell injury [29].

Studies focused on NGAL revealed high serum and urinary levels in patients with delayed graft function, defined as the need for dialysis in the first week after transplantation, concluding that urinary and serum NGAL measurement could be a promising biomarker in the early post-transplantation period [30-31]. A study performed on liver transplant recipients with Tac-induced AKI showed that urinary levels of NGAL at day 7 post surgery were significantly higher in patients with AKI as compared to the AKI-free group. This indicates that the urinary NGAL levels, measured at this particular time interval, can be a good predictive marker for Tac-induced AKI [32].

In our case, the elevated urinary markers were associated with acute Tac nephrotoxicity and not AR or delayed graft function as previous studies on kidney allografts pointed out. High NGAL values found in AKI secondary to Tac nephrotoxicity in liver transplanted patients are similarly to our findings. Thus, we could suggest that together, these urinary markers could be extremely useful in early diagnosing acute CNIs nephrotoxicity and differentiate it from acute rejection, but further studies are required.

To the best of our knowledge, this is the first case to report high values of KIM-1, NGAL, and cystatin $C$ associated with Tac nephrotoxicity.

\section{Conclusions}

The present data suggest that biomarkers like KIM-1, NGAL, cystatin C and IP-10, corroborated with common laboratory parameters prove to be critical tools in deciphering kidney graft associated pathology.

Extending such studies is of major importance as the identification and quantification of these markers in urine is a non-invasive approach that could finally lead to a very advantageous alternative to renal biopsy. 


\section{Acknowledgements}

This paper was published under the frame of European Social Fund, Human Resources Development Operational Program 2007-2013; project POSDRU/159/1.5/S/135760, CERO-Career Profile: Romanian Researcher.

\section{References}

1. Ogutmen B, Yildrim A, Sever MS, et al. Health related quality of life after kidney transplantation in comparison with intermittent hemodialysis, peritoneal dialysis, and normal controls. Transplant Proc 2006; 38(2):419-421.

2. Rabbat C, Kevine E, Thorpe J, Russel D, Churchill D. Comparison of mortality risk for dialysis patients and cadaveric first renal transplant recipients in Ontario, Canada. J Am Soc Nephrol 2000; 11:917-922.

3. Halawa A. The early diagnosis of acute renal graft dysfunction. A challenge we face. The role of novel biomarkers. Ann Transplant 2011; 16(1):90-98.

4. Srinivas $\mathrm{T}$, Meier-Kriesche $\mathrm{H}-\mathrm{U}$. Minimizing Immunosuppression, an alternative approach to reducing side effects: objectives and interim results. Clin J Am Soc Nephrol 2008; 3(Supll 2):S101-S116.

5. Lieberman KV, Lin WG, Reisman R. FK506 is a direct glomeruloconstrictor as determined by electrical resistant pulse sizing. Transplant Proc 1991; 23:3119-3120.

6. Bertram L, Kasiske L, Martin G, Zeier A, et al. KDIGO clinical practice guideline for the care of kidney transplant recipients: a summary. Kidney Int 2009.

7. Kjedsen L, Johnsen A, Sengelov H, Borregaard $\mathrm{N}$. Isolation and primary structure of NGAL, a novel protein associated with human neutrophil gelatinase. J Biol Chem 1993; 268:1042510432.

8. Ling $\mathrm{W}$, Zhaohui N, Ben H, Leyi G. Urinary IL18 and NGAL as early predictive biomarkers in contrast-induced nephropathy after coronary angiography. Nephron Clin Pract 2008; 108: 176-181.

9. Makris K, Markou N, Evodia E, et al. Urinary NGAL as an early marker of acute kidney injury in critically ill multiple trauma patients. Clin Chem Lab Med 2009; 47:79-82.

10. Ichimura $\mathrm{T}$, Bonventre JV, Bailly $\mathrm{V}$, et al. Kidney injury molecule-1(KIM-1), a Putative Epithelial Cell Adhesion Molecule Containing a novel immunoglobulin domain, is up-regulated in renal cells after Injury. J Biol Chem 1998; 273(7):4135-4142.
11. Huo W, Zhang K, Nie Z, Li Q, Jin F. Kidney injury molecule-1 (KIM-1): a novel kidneyspecific injury molecule playing potential double edged functions in kidney injury. Transplant Rev (Orlando) 2010; 23(3):143-146.

12. Liangos $\mathrm{O}$, Tighiouart $\mathrm{H}$, Perianayagam $\mathrm{M}$, et al. Comparative analysis of urinary biomarkers for early detection of acute kidney injury following cardiopulmonary bypass. Biomarkers 2009; 14(6):423-443.

13. Parikh CR, Mishra J, Thiessen-Philbrook $\mathrm{H}$, Dursun B, Ma Q, Kelly C, Dent C Devarajan P, Edelstein CL. Urinary II-18 is an early predictive biomarker in acute kidney injury after cardiac surgery. Kidney Int 2006; 70(1):199-203.

14. Booth V, Keizer DW, Kamphuis M, Clark-Lewis I, Sykes B. The CXCR3 binding chemokine IP10/CXCL10: structure and receptor interactions. Biochemistry 2002; 41(33):1041810425.

15. Jackson JA, Kim EJ, Begley B, Cheeseman J, et al. Urinary Chemokines CXCL9 and CXCL10 are non-invasive markers of renal allograft rejection and $\mathrm{BK}$ viral infection. $A m \mathrm{~J}$ Transplant 2011; 11(10):2228-2234.

16. Conti M, Moutereau S, Zater M, et al. Urinary cystatin $\mathrm{C}$ as a specific marker of tubular dysfunction. Clin Chem Lab Med 2006; 44(3):288-291.

17. Dumea R, Siriopol D, Hogas S, Mititiuc I, Covic A. Procalcitonin: diagnostic value in systemic infections in chronic kidney disease or transplant patients. Int Urol Nephrol 2014; 46(2):461-468.

18. Beqja-Lika A, Bulo-Kasneci A, Refatllari E, et al. Serum procalcitonine levels as an early diagnostic indicator of sepsis. Mat Soc Med 2013; 25(1):23-25.

19. Covic A. Nephrology. Theoretical and practical principles, $2^{\text {nd }}$ Edition. lasi: Demiurg 2011.

20. Kramer BK, Montagnino G, Del Castillo D, et al. Efficacy and safety of tacrolimus compared with cyclosporine A microemulsion in renal transplantation: 2 years follow- up results. Nephrol Dial Transplant 2005; 20(5):968-973.

21. Hardinger $K L$, Bohl DL, Schnitzler MA, et al. A randomized, prospective, pharmacoeconomic 
trial of tacrolimus versus cyclosporine in combination with thymoglobulin in renal

22. Knops N, Levtchenko E, Van den Heuvel B, Kuypers D. From gut to kidney: transporting and metabolizing calcineurin-inhibitors in solid organ transplantation. Int $J$ Pharm 2013; 452: 14-35.

23. Hocherl K, Dreher F, Vitzhum H, Kohler J, Kurtz A. Cyclosoprine A suppresses cyclooxygenase2 expression in the rat kidney. J Am Soc Nephrol 2002; 13:2427-2436.

24. Cortina $C$, Trojer $R$, Waldeger $S$, Schneeberger S, Gut N, Hofer J. De novo tacrolimus-induced thrombotic microangiopathy in the early stage after transplantation successfully treated with conversion to everolimus. Pediatric Nephrology 2015; 30(4):693-697.

25. Naesens m, Kuypers D, Sarwal M. Calcineurin Inhibitor Nephrotoxicity. Clin J Am Soc Nephrol 2009; 4:481-508.

26. Hall I, Koyner J, Doshi M, Marcus R, Parikh C. Urine Cystatin $C$ as a biomarker of proximal tubular function immediately after kidney transplantation. Am J Nephrol 2011; 33:407413.

27. Zhang PL, Rothblum LI, Han WK, Blasick TM, Potdar S, Bonventre JV. Kidney Injury Molecule-1 expression in transplant biopsies is transplant recipients. Transplantation 2005; 80: 41-46.

a sensitive measure of cell injury. Kidney Int 2008; 73(5):608-614.

28. Yadav B, Prasad N, Agrawal V, et al. Urinary kidney injury molecule-1 can predict delayed graft function in living donor renal allograft recipients. Nephrology (Carlton) 2015; 19.doi.10.1111

29. Song L, Xue L, Yu J, Zhao J, Zhang W, Fu Y. Kidney injury molecule-1 expression is closely associated with renal allograft damage. Bosn $\mathrm{J}$ Basic Med Sci 2013; 13(3):170-174.

30. Parikh CR, Jani A, Mishra J, et al. Urine NGAL and IL-18 are predictive biomarkers for delayed graft function following kidney transplantation. Am J Transplant 2006; 6:1639-1645.

31. Fonseca I, Oliveira JC, Almeida M, et al. Neutrophil Gelatinase-Associated Lipocalin in kidney transplantation is an early marker of graft dysfunction and is associated with one year renal function. Journal of Transplantation 2013;dx.doi.10.1155/650123.

32. Tsuchimoto $A$, Shinke $H$, Uesugi $M$, et al. Urinary Neutrophil Gelatinase-Associated Lipocalin; a useful biomarker for tacrolimusinduced acute kidney injury in liver transplant patients. Plos One 2014; 9(10):e110527. 